\section{Abatacept therapy in patients with JIA refractory to standard treatment}

Methotrexate, a DMARD used to treat rheumatoid arthritis, is also suitable for treating patients with juvenile idiopathic arthritis (JIA). Children who fail to respond to methotrexate can be treated with anti-tumor necrosis factor, although some do not respond or are intolerant to this line of therapy. Studies have shown that, in adults with rheumatoid arthritis, treatment with abatacept, a selective T-cell inhibitor, improves the condition. Ruperto and colleagues, therefore, investigated the effects of abatacept in children with JIA refractory to standard treatment.

Patients with JIA ( $n=190$, aged 6-17 years), recruited from medical centers in Europe, Latin America and the USA, received five doses of $10 \mathrm{mg} / \mathrm{kg}$ abatacept (30 min intravenous infusion) during an open-label period of 4 months. Those who responded positively were randomly assigned to receive either further treatment with abatacept $(n=60)$ or placebo $(n=62)$ at the point of randomization and every 28 days thereafter for 6 months, or until a disease flareup. More patients in the abatacept group had improved by at least $50 \%$ compared with controls $(77 \%$ vs $52 \%, P=0.0071)$. More flare-ups occurred in patients who were given placebo than in those who received abatacept (53\% vs $20 \%, P=0.0003)$. There was no significant difference in adverse events between the two groups. The authors noted that clinical improvements during the open-label treatment were maintained in many patients randomly assigned to receive placebo.

These results suggest that abatacept therapy is a suitable alternative for children with JIA who fail to respond or have intolerance to DMARDs.

Original article Ruperto N et al. (2008) Abatacept in children with juvenile idiopathic arthritis: a randomised, double-blind, placebo-controlled withdrawal trial. Lancet 372: 383-391

\section{Rivaroxaban prevents blood clots after major orthopedic surgery}

Rivaroxaban, an inhibitor of factor $\mathrm{Xa}$, has demonstrated superior efficacy to enoxaparin, a low-molecular-weight heparin, in the prevention of venous thrombosis after joint replacement. The results have been published in three phase III clinical trials carried out as part of the RECORD studies (regulation of coagulation in major orthopedic surgery reducing the risk of deep vein thrombosis and pulmonary embolism).

In RECORD 1 and 3, major venous thromboembolism occurred in fewer patients treated with rivaroxaban (10 mg once daily) than in patients treated with enoxaparin $(40 \mathrm{mg}$ once daily) after hip ( $0.2 \%$ vs $2 \%$ ) or knee replacement (1.0\% vs $2.6 \%)$. RECORD 2 revealed, at the same doses, long-term treatment with rivaroxaban (31-39 days) to be significantly more effective than short-term treatment with enoxaparin (10-14 days) for the prevention of major venous thromboembolism, with an incidence of $0.6 \%$ and $5.1 \%$, respectively, after total hip arthroplasty.

As venous thromboembolism is a serious complication of major orthopedic surgery, extended thromboprophylaxis is recommended. Enoxaparin must be administered subcutaneously, which the patient or caregiver must be trained to perform. Rivaroxaban is a potentially ideal alternative as it is taken orally, making extended therapy easier to manage, and has comparable efficacy and safety profiles to enoaxaparin.

\footnotetext{
Original articles Eriksson BI et al. (2008) Rivaroxaban versus enoxaparin for thromboprophylaxis after hip arthroplasty. N Engl J Med 358: 2765-2775

Kakkar AK et al. (2008) Extended duration rivaroxaban versus short-term enoxaparin for the prevention of venous thromboembolism after total hip arthroplasty: a doubleblind, randomised controlled trial. Lancet 372: 31-39 Lassen MR et al. (2008) Rivaroxaban versus enoxaparin for thromboprophylaxis after total knee arthroplasty. N Engl J Med 358: 2776-2786
}

\section{Daily alendronate sodium is better tolerated than once-weekly treatment for Paget disease}

Alendronate sodium is a bisphosphonate used to treat Paget disease of bone (PDB). The approved dosage of $40 \mathrm{mg} /$ day for 6 months is generally well tolerated, but esophageal irritation can occur if the tablet is not delivered directly to the stomach. Increasing the dose and delivering the drug less frequently could reduce the incidence of upper gastrointestinal adverse events and might improve adherence. 